\title{
Diabetic Retinopathy and Laser Therapy in Rats: A Protein-Protein Interaction Network Analysis
}

\author{
Akram Safaei', Mostafa Rezaei Tavirani', Mona Zamanian Azodi ${ }^{1}$, Alireza Lashay ${ }^{2}$, Seyed Farzad \\ Mohammadi $^{2}$, Mohamad Ghasemi Broumand ${ }^{3}$, Ali Asghar Peyvandi ${ }^{4}$, Farshad Okhovatian ${ }^{3 *}$, Hassan \\ Peyvandi ${ }^{4}$, Mohammad Rostami Nejad ${ }^{5}$ \\ ${ }^{1}$ Proteomics Research Center, Shahid Beheshti University of Medical Sciences, Tehran, Iran \\ ${ }^{2}$ Eye Research Center, Farabi Eye Hospital, Tehran University of Medical Sciences, Tehran, Iran \\ ${ }^{3}$ Physiotherapy Research Centre, School of Rehabilitation, Shahid Beheshti University of Medical Sciences, Tehran, Iran \\ ${ }^{4}$ Hearing Disorder Research Center, Shahid Behshti University of Medical Sciences, Tehran, Iran \\ ${ }^{5}$ Gastroenterology and Liver Diseases Research Center, Research Institute for Gastroenterology and Liver Diseases, Shahid \\ Beheshti University of Medical Sciences, Tehran, Iran
}

\author{
*Correspondence to \\ Farshad Okhovatian, PhD; \\ Physiotherapy Research Centre, \\ School of Rehabilitation, Shahid \\ Beheshti University of Medical \\ Sciences, Tehran, Iran. \\ Tel: +98-2 2721174 ; \\ Fax: +98-21 22721174 \\ Email: \\ farshadokhovatian1965@gmail.com
}

Published online 29 August 2017

\begin{abstract}
Introduction: Diabetic retinopathy (DR) is a serious microvascular complication of diabetes which can cause vision loss or blindness ultimately. Non enzymatic glycation of proteins leads to advanced glycation end products (AGEs) in DR. Since laser therapy is a well-established method, in this study, protein-protein interaction (PPI) network is applied for protein targets in DR disease in rats treated by laser.

Methods: In this study, we focused on articles that investigated and compared the proteome profiles of DR rats with healthy control and also DR rats before and after laser therapy. The networks of related differentially expressed proteins were explored using Cytoscape version 3.3.0, the PPI analysis methods and ClueGO.

Results: Analysis of PPI network of 37 related proteins to DR rats including 108 nodes, introduced 10 hub-bottleneck proteins and 5 concerned biochemical pathways. On the other hand, PPI analysis of related proteins to DR rats before and after laser therapy corresponded to 33 proteins and 2 biological pathways.

Discussion: Centrality and cluster screening identified hub-bottelneck genes, including Aldoa, HSPD1, Pgam2, Mapk3, SLC2A4, Ctnnb1, Ywhab, HSPA8, GAPDH and Actb for DR rats versus healthy control and ENO1, Aldoa, GAPDH for DR samples after laser therapy.

Conclusion: Gene expression analysis of the DR samples treated via laser therapy provides a molecular evidence in support of the therapeutic effect of laser.

Keywords: Diabetic retinopathy rat; Laser therapy; Protein-protein interaction network (PPI) analysis.
\end{abstract}

\begin{abstract}
Introduction
Diabetic retinopathy (DR) is a serious microvascular complication of diabetes which leads to vision loss or blindness ultimately. In fact, it is one of the most frequent causes of visual impairment in the world. ${ }^{1}$ Signs of DR are retinal vascular permeability and retinal ischemia. Since progression of DR are accompanied with biochemical, cellular and histopathological changes that are not detectable by the clinician, molecular investigation is an important requirement. ${ }^{2}$ It is reported that in DR genetic, environmental, and immunological factors act together. ${ }^{3,4}$ The main process affected in patients is hyperglycemia. Chronic high levels of blood glucose in diabetic patients effect destructively on retina because retina cells are sensitive to overflow of glucose. One of the complications of DR is vascular permeability changes
\end{abstract}

which plays an important role in the development of visual impairment in diabetes. Non enzymatic glycation of proteins leads to advanced glycation end products (AGEs). AGEs are able to produce cross-links between proteins. ${ }^{5,6}$ In vitro and in vivo investigation have proven the relationship between AGEs concentration and DR signs. ${ }^{7}$ Diabetic condition induces some alterations in the expression of a diverse range of retinal proteins, so study of biochemical pathways may provide valuable insights into the pathogenesis of DR. ${ }^{8}$ Protein complexes are known as essential components in cellular processes and analysis of the proteins in these complexes is a state of art approach. ${ }^{9}$ Protein- protein interaction network analysis in pathophysiological conditions attracted significant attention from researchers, in order to discover an integrated biological output. ${ }^{10}$ Human molecular 
interaction networks are not just basic science exercise for a more clear view of fundamental human biology but will transform molecular diagnosis, treatment and patients monitoring tools. ${ }^{11-13}$ Protein networks analysis provides a scientific model that can improve understanding of the mechanisms underlying human diseases. ${ }^{14-19}$ Pathogenesis of DR is complex, and despite a lot of research, it has not been completely elucidated. Published evidence supported clinical use of lasers for the proliferative DR macular edema. ${ }^{20}$ The importance of early treatment of DR depends on the level of knowledge in molecular changes and cellular processes. ${ }^{21,22}$ Identification and comparison of the protein-protein interaction (PPI) changes and involved pathways in the diabetic retina before and after laser will provide clues to the underlying therapeutic mechanism(s) involved in laser treatment as well as other diseases. ${ }^{17,18,23}$ Laser therapy is used as an anti-VEGF treatment of DR patients but this therapeutic method may cause retinal damage and scarring. ${ }^{24}$ Discovery of molecular interventions and understanding disease processes can be helpful in introducing less invasive therapeutic options. In this study, we want to analyze PPI network to identify critical proteins and signaling pathways related to diabetic nephropathy and also in response to laser therapy. It is aimed to reveal common molecular changes in DR pathogenesis and following laser therapy.

\section{Methods}

Selection of Differentially Expressed Genes

The PubMed database and Google Scholar were cross-searched (on June 2015) for the terms "diabetic retinopathy" ,"proteomic", "laser", and "treatment" (i.e., title, abstract, keyword). A total of 25 research papers were collected which surveyed tissues, serum, plasma, saliva and urine. Among them, studies with focus on same organism and same cell-position before and after treatment were selected. Finally, a study that had surveyed proteome map of normal rat retina in comparison with the proteome of diabetic rat retina was selected ${ }^{25}$ (Table 1) and another one focusing on protein changes in rats after laser treatment ${ }^{26}$ (Table 2).

Construction and Topological Analysis of PPI Networks PPI networks were generated using Cytoscape version 3.3.0. ${ }^{27}$ The advantage of Cytoscape is that users can analyze and visualize networks of genes and compounds, and identify enriched pathways from expression profiling data. Two centrality parameters such as degree and betweenness were considered to determine the hub and bottleneck proteins. First, we prepared the list of proteins which were hub in PPI network of DR versus controls in the PPI network. The nodes with high degree defined as hub proteins and the nodes with high betweenness defined as bottleneck proteins, which both play pivotal roles in the network. ${ }^{28}$ The results indicate that some hub nodes play a role as bottleneck, these proteins were introduced
Table 1. Thirty-Seven Changed Expression Proteins in Retina of Rats With Diabetic Retinopathy in Comparison With Healthy Control Rats

\begin{tabular}{|c|c|}
\hline Protein Name & Gene Name \\
\hline Enolase 2 gamma & ENO2 \\
\hline Heat shock protein $701 \mathrm{~A}$ & HSPA1A \\
\hline Heat shock protein 8 & HSPA8 \\
\hline Aldehyde reductase 1 & AKR1B1 \\
\hline Triose phosphate isomerase & TPI1 \\
\hline Phosducin & PDC \\
\hline 14-3-3 protein & Ywhab \\
\hline Crystallin B1 & CRYBB1 \\
\hline Beta catenin complex B & Ctnnb1 \\
\hline Creatine kinase B & CKB \\
\hline Glyceraldehyde-3-phosphate dehydrogenase & GAPDH \\
\hline Enolase1 & ENO1 \\
\hline Beta actin & Actb \\
\hline Malate dehydrogenase & Mdh \\
\hline Dimethylarginine dimethlaminohydrolase & Ddah1 \\
\hline Calreticulin & Calr \\
\hline Proteasome alpha 6 subunit & Psma6 \\
\hline Phosphoglycerate mutase & Pgam \\
\hline Aminoacyclase 1 & Acy1a \\
\hline Succinyl CoA ligase ADP forming subunit & Suclg1 \\
\hline Dystrophin-related protein 2 & DRP 2 \\
\hline Inositol 1 monophosphate & Impa1 \\
\hline Triosephosphate isomerase & Tpi1 \\
\hline Chaperonin subunit 2 & Hspd1 \\
\hline ATP synthase B subunit & Atp5b \\
\hline Tubulin beta & TUBB3 \\
\hline Histone 2B & Hist1h2ba \\
\hline ATP-ase, vesicle fusing & Nsf \\
\hline Pyridoxine kinase & Pdxk \\
\hline Calbindin & Calb1 \\
\hline Pyruvate dehydrogenase & Pdk2 \\
\hline Crystatin B & Cstb \\
\hline Profilin 2 & Pfn2 \\
\hline Glutamate ammonia ligase & Glul \\
\hline Fructose bisphosphate aldolase A & Aldoa \\
\hline Superoxide dismutase & Sod1 \\
\hline Isovaleryl coenzyme A dehydrogenase & Ivd \\
\hline
\end{tabular}

as hub-bottleneck. ClueGO was used for data analysis. The degree of functional enrichment for a given cluster was quantitatively assessed (statistical significance was calculated) using the ClueGO tool. ${ }^{29}$ ClueGO integrates gene ontology $(\mathrm{GO})$ terms and creates a functionally organized $\mathrm{GO} /$ pathway term network. It can analyze genes and comprehensively visualize functionally grouped terms. ${ }^{29}$ A pack of gene annotations (e.g. functions, processes) can help identify interesting features, but due to the huge size of the sets, selecting statistically significant trends from large datasets is impractical. Thus, a method is required for the routine analysis of such datasets. We used GO as a common vocabulary for annotation as it 
Table 2. A Number of Changed Expression Proteins in Retina of Rats With Diabetic Retinopathy Before and After Laser Therapy

\begin{tabular}{lc}
\hline Protein Name & Gene Name \\
\hline Alpha-A crystalline & Cryaa \\
Recoverin & Rcvrn \\
Isovaleryl coenzyme A dehydrogenase & Ivd \\
Claudin-12 & Cldn12 \\
Enolase 1 alpha & Eno1 \\
Germinal histone H4 & Hist1h4b \\
Glyceraldehyde 3 phosphate dehydrogenase & Gapdh \\
Aldolase A & Aldoa \\
Wnt-5 beta & Wnt5a \\
LEK-1 & Cenpf \\
Dismutase & Sod \\
Calretinin & Calb2 \\
\hline
\end{tabular}

allows identification of semantically related genes and gene products. ${ }^{30}$ Kappa statistic $\geq 0.4$, enrichment and Bonferroni step down method were used for probability value correction. ${ }^{31}$

Laser Parameters

The laser (20 spots of $100-\mathrm{mW}$ power, $200-\mu \mathrm{m}$ diameter, $0.1 \mathrm{~ms}$ ) which has been used in rat DR studies was Argon green with a wavelength of $514 \mathrm{~nm}$ slit-lamp laser (HGM Medical Laser Systems Inc., USA). The one eye of the rat DR samples was treated by laser under anaesthesia condition. ${ }^{26}$

\section{Results}

Topological Analysis of PPI Networks for Diabetic Retinopathy vs. Normal Rats

Cytoscape analysis revealed a great number of close interconnections (see Figure 1). The PPI network properties are tabulated in Table 3 . The top $10 \%$ proteins in terms of degree and betweenness (2) were defined as

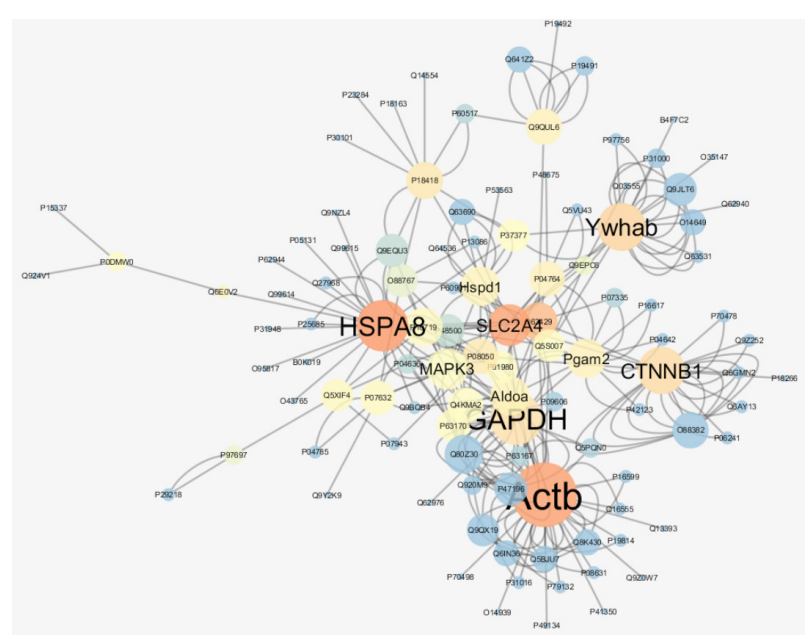

Figure 1. PPI network which consists of 108 nodes and 56 multi-edge node pairs for diabetic retinopathy versus normal rats. The larger circles correspond to the higher degree and blue to brown color refers to increment of betweenness value
Table 3. Properties of protein-protein interaction Network of Diabetic Retinopathy Versus Normal Rats

\begin{tabular}{lc}
\hline Topological Parameters & Values \\
\hline Number of nodes & 108 \\
Multi-edge node pairs & 56 \\
Average degree & 4.981 \\
Average betweenness & 0.023 \\
Average closeness centrality & 0.309 \\
Network density & 0.029 \\
\hline
\end{tabular}

Table 4. The Hub-Bottleneck Nodes in the Protein Network of Diabetic Retinopathy Versus Normal Rats

\begin{tabular}{lccc}
\hline Gene Name & Degree & Betweenness & Uniprot Code \\
\hline Aldoa & 14 & 0.055213 & P05065 \\
HSPD1 & 14 & 0.055388 & P63039 \\
Pgam2 & 14 & 0.060204 & P16290 \\
Mapk3 & 16 & 0.030572 & P21708 \\
SLC2A4 & 17 & 0.297383 & P19357 \\
Ctnnb1 & 24 & 0.133282 & Q9WU82 \\
Ywhab & 25 & 0.153595 & P35213 \\
HSPA8 & 29 & 0.31212 & P63018 \\
GAPDH & 30 & 0.11042 & P04797 \\
Actb & 46 & 0.30541 & P60711 \\
\hline
\end{tabular}

hubs and bottlenecks respectively. These key proteins are tabulated in Table 4. As it is depicted in Table 4 all hub proteins play a role as bottlenecks too; so we introduced them as hub-bottleneck proteins. The GO pathway clusters enriched by crucial proteins in DR rats versus healthy controls are shown in Table 5.

PPI Network Analysis for Diabetic Retinopathy Rats Before and After Laser Therapy

The results of network analysis in subpart 3.1 and the results of PPI network analysis related to DR rats before and after laser therapy are shown in Figure 2 and Tables 6-8.

\section{Discussion}

As mentioned in Methods, 37 differentially expressed proteins are associated with DR rats (disease state). However, 12 proteins differentiate the treated samples from DR rats (treated state). The information is tabulated in Tables 1 and 2. Furthermore, networks corresponding to disease and treated states are analyzed and presented in Figures 1 and 2. As illustrated in these figures, disease state network consists of 108 nodes while treated state network includes 33 nodes. Both networks are about three folds wider than the initial proteins. In addition, about half of the nodes of the 2 networks are multi-edges. At the first glance, as presented in Tables 3 and 6, on average the 2 networks are $40 \%$ different. However, based on average betweenness, the networks are 5 folds dissimilar. Other 
Table 5. The Concerned Pathways of Differentially Expressed Proteins in Diabetic Retinopathy Rats Versus Normal Group $(P<0.05)$

\begin{tabular}{lllll}
\hline $\mathbf{R}$ & GO ID & GO Term & No. & Associated Gene Found \\
\hline 1 & GO:0010226 & Response to lithium ion & 3 & Calr, impa1, ctnnb1 \\
2 & GO:0046686 & Response to cadmium ion & 3 & Sod1, hspa8, ctnnb1 \\
3 & GO:0050821 & Protein stabilization & 4 & Calr,pfn2, hspa1b, gapdh \\
4 & GO:0009408 & Response to heat & 4 & Hsp1, hspa8, calr, hspa1b \\
5 & GO:0072524 & $\begin{array}{l}\text { Pyridine-containing compound } \\
\text { metabolic process }\end{array}$ & 13 & $\begin{array}{l}\text { Impa1, hspa1b, akr1b1, atp5b, pdk2, gapdh, eno2, aldoa, pgam2, tpi1, } \\
\text { eno1, mdh2, qdxk }\end{array}$ \\
\hline
\end{tabular}

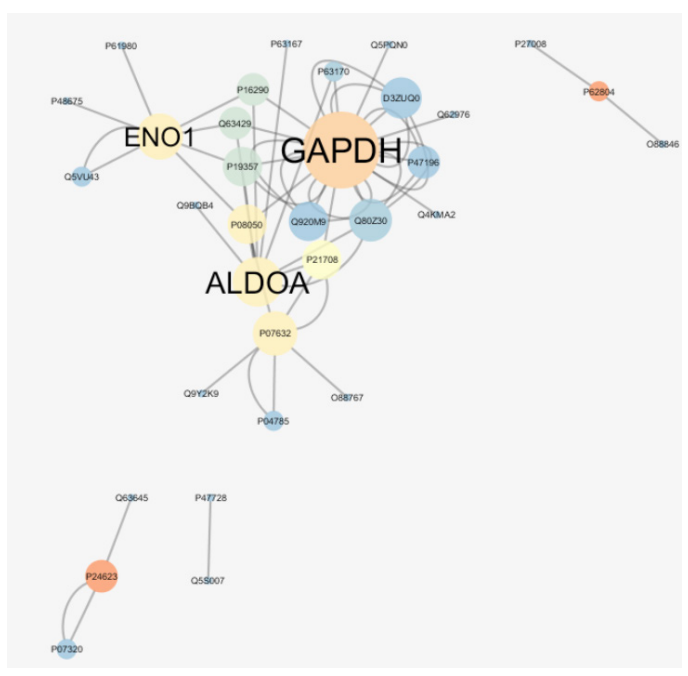

Figure 2. PPI Network With 33 Nodes and 14 Multi-Edge Node Pairs in Diabetic Retinopathy Rats Before and After Laser Therapy. The larger circles correspond to the higher degree and blue to brown color refers to increment of betweenness value.

Table 6. Properties of PPI Network of Diabetic Retinopathy Rats Before and After Laser Therapy

\begin{tabular}{lc}
\hline Topological Parameters & Values \\
\hline Number of nodes & 33 \\
Multi-edge node pairs & 14 \\
Average degree & 3.43 \\
Average betweenness & 0.104 \\
Average closeness centrality & 0.497 \\
Network density & 0.074 \\
\hline
\end{tabular}

Table 7. The Key Nodes (Hub-Bottleneck Proteins) of PPI Network of Diabetic Retinopathy Rats Before and After Laser Therapy

\begin{tabular}{llll}
\hline Gene Name & Degree & Betweenness Centrally & Uniprot Code \\
\hline ENO1 & 9 & 0.266403 & P04764 \\
ALDOA & 14 & 0.264471 & P05065 \\
GAPDH & 30 & 0.489767 & P04797 \\
\hline
\end{tabular}

Table 8. Pathways of Differentially Expressed Proteins in Diabetic Retinopathy Rat Before and After Laser.

\begin{tabular}{llll}
\hline $\mathbf{R}$ & GO Term & No. & Associate Genes Found \\
\hline 1 & $\begin{array}{l}\text { Columnar/cuboidal epithelial } \\
\text { cell development }\end{array}$ & 2 & Wnt5a, sod1 \\
2 & Pyruvate metabolic process & 3 & Gapdh, Endo1, Aldoa \\
\hline
\end{tabular}

topological parameters of these networks, such as average closeness centrality and average shortest path length were calculated and shown in Tables 3 and 6. Graph centrality properties include essential parameters like degree, betweenness and closeness centrality in a PPI network. ${ }^{32}$ One of the important properties of a network is the degree centrality that is related to the node connectivity (the number of connections of a node). A node with high degree value is known as a hub node. Another important component in network analysis is betweenness, which is one of the most important topological properties of a network. Bottlenecks that are key connector proteins have been introduced as proteins with a high betweenness centrality (nodes that many "shortest paths" pass through them).$^{28}$ In system biology, there are some researches that have focused on hubs while others have argued bottlenecks are more crucial proteins. ${ }^{28}$ Therefore, we further focused on both features of high degree and high betweenness to obtain a better predictor of protein essentiality in both regulatory and interaction networks. The topologically significant nodes for both networks were extracted as hubs and bottlenecks as the proteins that were in the top $10 \%$ in both terms of degree and betweenness centrality. The determined hubs and bottlenecks for the two states are shown in Tables 4 and 7. There are 10 and 3 hub and bottleneck proteins for $\mathrm{RD}$ and treated states respectively. As shown in Tables 4 and 7 the hub proteins are also bottlenecks. The introduced hub-bottleneck proteins for disease state are Aldoa, HSPD1, Pgam2, Mapk3, SLC2A4, Ctnnb1, Ywhab, HSPA8, GAPDH and Actb. ALDOA is a key enzyme in glycolysis and acts in regulation of cell proliferation and actin cytoskeleton organization. ${ }^{33,34}$ It is reported that the anti-aldolase autoantibody serves as a useful marker for DR diagnosis. ${ }^{35}$ HSPD1 or HSP60 plays a key role in preventing apoptosis as well as immune response. ${ }^{36}$ Expression of HSPD1 increases in dry eye. ${ }^{37}$ Differential gene expression of HSPD1 in retinoblastoma compared to normal retina is reported. ${ }^{38}$ In addition, age-related changes in expression of heat-shock proteins, including HSPD1 is reported in rats. ${ }^{39}$ PGAM is a glycolytic enzyme that catalyzes the reversible conversion of 3-PGA to 2-PGA. ${ }^{40}$ This enzyme is up regulated in many human cancers. ${ }^{41}$ However, there was no obvious evidence of its relation with retinopathy until now. Mapk3 acts in a signaling cascade that regulates various cellular processes such as proliferation and cell cycle. ${ }^{42}$ Alteration 
in activation of Mapk3 influences cancer development. ${ }^{43,44}$ This enzyme is involved in the release of VEGF in diabetic rat retina; therefore, it may be a potential therapeutic target of DR. ${ }^{45} \mathrm{Ctnnb} 1$ encodes $\beta$-catenin and regulates the coordination of cell-cell adhesion and gene transcription ${ }^{46}$ as well as acting as an intracellular signal transducer in the Wnt signaling pathway. ${ }^{47}$ Its abnormal expression leads to various diseases including cancer. ${ }^{48}$ $\mathrm{WNT} / \beta$-catenin signaling also is introduced as a new link between diabetes and cancer. ${ }^{49,50} \mathrm{~A}$ role for $\beta$-catenin in glucose and energy homeostasis is reported. ${ }^{51}$ Inhibition of connective tissue growth factor overexpression in DR via blocking the WNT/beta-catenin pathway confirms the association of beta-catenin with DR. ${ }^{52}$ Ywhab encodes a protein that is involved in signal transduction and plays a role in cell cycle. ${ }^{53}$ This protein as a member of the 14-3-3 protein family plays a key role in cellular proliferation and development of breast cancer. ${ }^{54}$ The report of 14-3-3 proteins relationship with DR is limited to a study that shows expression change in early diabetic rat retinal proteomes versus normal. ${ }^{25}$ Chaperones like HSPA8 increase cell survival ${ }^{55}$ and play a key role in the maintenance of epithelial cell structure and function and are also responsible for cell repair processes after damage, proliferation, apoptosis and modulate cell signaling. ${ }^{56}$. HSPA8 protective role was further highlighted in a study that identified HSPA8 alongside other HSP70 proteins suppressed aging brains. ${ }^{57}$ Actin, cytoplasmic 1 with highest score among the hub-bottleneck proteins has a conserved gene in eukaryotes, which play an essential role in retina development. ${ }^{58}$ Glyceraldehyde-3-phosphate dehydrogenase (GADPH) is a critical enzyme in glucoseinduced apoptosis of retinal Muller cells. ${ }^{59}$ Moreover, the role of GADPH in the development and progression of DR was investigated by Kanwar and Kowluru. ${ }^{60}$ Regulation of actin-based motility by Rho was introduced as an important pathway according to analysis of the DR saliva. ${ }^{61}$ ENO1 is a hub-bottleneck in treated network. Its overexpression has been associated with multiple tumors. $^{62-64}$ In many of these tumors, ENO1 promoted tumorgenesis via activating and regulating the PI3K/ AKT signaling pathway. ${ }^{63}$ It is shown that the knockdown of ENO1 expression led to suppressed cell growth and migration by inactivating the PI3K/Akt pathway in glioma cells. ${ }^{63}$ Tear proteomic analysis of patients with type 2 diabetes with dry eye syndrome shows alteration in expression of ENO1 ${ }^{65}$ as well as results on DR in rat. ${ }^{25}$ Another hub-bottleneck after laser therapy is ALDOA, which can be suggested as a common hub-bottleneck for DR progression and laser therapy. It is introduced as a potential metastasis-associated marker of lung squamous cell carcinoma. ${ }^{66}$ Aldolases $\mathrm{A}$ and $\mathrm{C}$ are mainly involved in glycolysis ${ }^{67}$ and glycolysis itself has been reported to be increased in DR. ${ }^{68}$ Some data demonstrate that the anti-aldolase autoantibody can serve as a useful marker for DR diagnosis because of high level in serum ${ }^{35}$; interestingly, following laser treatment the ALDOA expression decreased in DR. ${ }^{26}$ The alteration of glycolysis in laser therapy of diabetic retina may reflect metabolic improvement caused by laser treatment. GAPDH is another common hub-bottleneck protein for the 2 networks, which has highest centrality score. GAPDH is the key glycolytic enzyme. Increase in glycolysis, specially overexpression of GAPDH is considered as an essence of many types of cancer. ${ }^{69}$ Non-glycolytic role of GAPDH in cancer by interacting with telomerase RNA component is reported.$^{70}$ Diacylglycerol (DAG) is a key activator of protein kinase $\mathrm{C}$, it increases glycolysis and endothelial cell proliferation as well as endothelial permeability. ${ }^{71,72}$ Down regulation of aldolase A and GAPDH after laser treatment ${ }^{26}$ can control the hyper activity of glycolysis which occurs in DR patients. ${ }^{68}$ Agents that regulate GAPDH can be potential candidates in inhibiting the progression of DR. ${ }^{73}$ In this study, we introduced 3 hubbottleneck proteins that are involved in laser treated DR rats. Two of these key proteins (66\%) are in common with the central proteins that differentiate DR rats from the healthy groups; so, it can be concluded that the treated are approximately normalized towards the healthy rats.

The GO pathway clusters enriched by crucial proteins in DR rats versus healthy controls (see Table 5) provide a series of candidates for mechanism research, such as response to lithium ion, response to cadmium ion, protein stabilization, and response to heat and pyridinecontaining compound. Their role is plausible as most of the enriched pathways including protein stabilization, and response to heat pathways were previously shown to be associated with DR. ${ }^{74-76}$ The related GO pathway clusters after laser therapy are determined as columnar/ cuboidal epithelial cell development and pyruvate metabolic process (see Table 8). DR involves an abnormal pathology of major retinal cells, including retinal pigment epithelium, inter-retinal edema, microaneurysms. ${ }^{77}$ So involving the pathway in epithelial development such as columnar/cuboidal epithelial cell development is not unexpected. This process is involved in most of the mentioned characteristic features of $\mathrm{RD}$ and the introduced crucial proteins are attributed in regulation of this process. Pyruvate metabolic process is another detected pathway. Pyruvate is the end-product of glycolysis $^{78}$ and its dysmetabolism contributes to failure of the pancreatic islet $\beta$-cells during late type 2 diabetes. ${ }^{79}$ Its up regulation can lead to excessive fatty acid oxidation and ROS formation in the mitochondria. ${ }^{80}$

\section{Conclusion}

Aldoa, HSPD1, Pgam2, Mapk3, SLC2A4, Ctnnb1, Ywhab, HSPA8, GAPDH and Actb are the 10 key proteins in PPI network of DR rats. However ENO1, ALDOA and GAPDH are the 3 central proteins related to treatment of DR rats by laser. This provides molecular evidence in support of therapeutic effect of laser.

\section{Conflict of Interests}

None. 


\section{Ethical Considerations}

Not applicable.

\section{Acknowledgments}

This research was supported by Shahid Beheshti University of Medical Sciences.

\section{References}

1. Cade WT. Diabetes-related microvascular and macrovascular diseases in the physical therapy setting. Phys Ther. 2008;88(11):1322-35. doi: 10.2522/ptj.20080008.

2. Grunwald JE, DuPont J, Riva CE. Retinal haemodynamics in patients with early diabetes mellitus. Br J Ophthalmol. 1996;80(4):327-31.

3. Zorena K, Raczyńska D, Raczyńska K. Immunological Risk Factors for the Development and Progression of Diabetic Retinopathy. INTECH; 2012.

4. Abu El-Asrar AM, Nawaz MI, Kangave D, Mairaj Siddiquei $\mathrm{M}$, Geboes K. Angiogenic and vasculogenic factors in the vitreous from patients with proliferative diabetic retinopathy. J Diabetes Res. 2013;2013:539658. doi: 10.1155/2013/539658.

5. Bierhaus A, Hofmann MA, Ziegler R, Nawroth PP. AGEs and their interaction with AGE-receptors in vascular disease and diabetes mellitus. I. The AGE concept. Cardiovasc Res. 1998;37(3):586-600.

6. Goh SY, Cooper ME. Clinical review: The role of advanced glycation end products in progression and complications of diabetes. J Clin Endocrinol Metab. 2008;93(4):1143-1152. doi: 10.1210/jc.2007-1817.

7. Zorena K, Raczynska D, Raczynska K. Biomarkersin diabetic retinopathy and the therapeutic implications. Mediators Inflamm. 2013;2013:193604. doi: 10.1155/2013/193604.

8. Umadevi S, Premkumar K, Valarmathi S, Ayyasamy PM, Rajakumar S. Identification of novel genes related to diabetic retinopathy using protein-protein interaction network and gene ontologies. J Biol Syst. 2016;24(01):11727. doi: 10.1142/S0218339016500066.

9. Abbaszadeh H-A, Peyvandi AA, Sadeghi Y, et al. Er: YAG laser and cyclosporin A effect on cell cycle regulation of human gingival fibroblast cells. J Lasers Med Sci. 2017;8(3):143-149. 10.22037/2010.v8i3.16363.

10. Ivanov AA, Khuri FR, Fu H. Targeting protein-protein interactions as an anticancer strategy. Trends Pharmacol Sci. 2013;34(7):393-400. doi: 10.1016/j.tips.2013.04.007.

11. Ideker T, Sharan R. Protein networks in disease. Genome Res. 2008;18(4):644-52. doi: 10.1101/gr.071852.107.

12. Zamanian-Azodi M, Rezaei-Tavirani M, Nejadi N, et al. Serum proteomic profiling of obsessive-compulsive disorder, washing subtype: a preliminary study. Basic Clin Neurosci. 2017;8(4):307-316.

13. Zamanian-Azodi M, Rezaei-Tavirani M, Rahmati-Rad S, Rezaei Tavirani M. Ethanol and cancer induce similar changes on protein expression pattern of human fibroblast cell. Iran J Pharm Res. 2016;15(Suppl):175-184.

14. Goh K-I, Cusick ME, Valle D, Childs B, Vidal M, Barabási A-L. The human disease network. Proceedings of the National Academy of Sciences. 2007;104(21):8685-8690. doi:10.1073/pnas.0701361104.

15. Yang X, Deignan JL, Qi H, et al. Validation of candidate causal genes for obesity that affect shared metabolic pathways and networks. Nat Genet. 2009;41(4):415-423.
16. Safari-Alighiarloo N, Taghizadeh M, Rezaei-Tavirani M, Goliaei B, Peyvandi AA. Protein-protein interaction networks (PPI) and complex diseases. Gastroenterol Hepatol Bed Bench. 2014;7(1):17-31.

17. Safaei A, Rezaei Tavirani M, Arefi Oskouei A, Zamanian Azodi M, Mohebbi SR, Nikzamir AR. Protein-protein interaction network analysis of cirrhosis liver disease. Gastroenterol Hepatol Bed Bench. 2016;9(2):114-123.

18. Zamanian-Azodi M, Rezaei-Tavirani M, Rahmati-Rad S, Hasanzadeh H, Rezaei Tavirani M, Seyyedi SS. ProteinProtein Interaction Network could reveal the relationship between the breast and colon cancer. Gastroenterol Hepatol Bed Bench. 2015;8(3):215-224.

19. Rezaei-Tavirani M, Zamanian-Azodi M, Rajabi S, Masoudi-Nejad A, Rostami-Nejad M, Rahmatirad S. Protein clustering and interactome analysis in Parkinson and Alzheimer's diseases. Arch Iran Med. 2016;19(2):101109.

20. Neubauer AS, Ulbig MW. Laser Treatment in Diabetic Retinopathy. Ophthalmologica. 2007;221(2):95-102.

21. Kalantari S, Rutishauser D, Samavat S, et al. Urinary prognostic biomarkers and classification of IgA nephropathy by high resolution mass spectrometry coupled with liquid chromatography. PLoS One. 2013;8(12):e80830. doi: 10.1371/journal.pone.0080830.

22. Zali H, Rezaei-Tavirani M, Kariminia A, Yousefi R, Shokrgozar MA. Evaluation of growth inhibitory and apoptosis inducing activity of human calprotectin on the human gastric cell line (AGS). Iran Biomed J. 2008;12(1):714.

23. Zali H, Rezaei Tavirani M. Meningioma protein-protein interaction network. Arch Iran Med. 2014;17(4):262-272.

24. Marshall J. Thermal and mechanical mechanisms in laser damage to the retina. Invest Ophthalmol. 1970;9(2):97-115.

25. Quin G, Len AC, Billson FA, Gillies MC. Proteome map of normal rat retina and comparison with the proteome of diabetic rat retina: new insight in the pathogenesis of diabetic retinopathy. Proteomics. 2007;7(15):2636-2650. doi: 10.1002/pmic.200600486.

26. Quin GJ, Lyons B, Len AC, Madigan MC, Gillies MC. Proteome changes induced by laser in diabetic retinopathy. Clin Exp Ophthalmol. 2015;43(2):180-187. doi: 10.1111/ ceo.12372.

27. Shannon P, Markiel A, Ozier O, et al. Cytoscape: a software environment for integrated models of biomolecular interaction networks. Genome Res. 2003;13(11):24982504. doi: 10.1101/gr.1239303.

28. Yu H, Kim PM, Sprecher E, Trifonov V, Gerstein M. The importance of bottlenecks in protein networks: correlation with gene essentiality and expression dynamics. PLoS Comput Biol. 2007;3(4):e59. doi: 10.1371/journal. pcbi.0030059.

29. Bindea G, Mlecnik B, Hackl H, et al. ClueGO: a Cytoscape plug-in to decipher functionally grouped gene ontology and pathway annotation networks. Bioinformatics. 2009;25(8):1091-3. doi: 10.1093/bioinformatics/btp101.

30. Liu $\mathrm{H}, \mathrm{Hu} \mathrm{ZZ}$, Wu CH. DynGO: a tool for visualizing and mining of Gene Ontology and its associations. BMC Bioinformatics. 2005;6:201. doi: 10.1186/1471-2105-6-201.

31. Rivera CG, Vakil R, Bader JS. NeMo: Network Module identification in Cytoscape. BMC Bioinformatics. 2010;11 Suppl 1:S61. doi: 10.1186/1471-2105-11-s1-s61.

32. Khuri S, Wuchty S. Essentiality and centrality in protein 
interaction networks revisited. BMC Bioinformatics. 2015;16:109. doi: 10.1186/s12859-015-0536-X.

33. Yao DC, Tolan DR, Murray MF, et al. Hemolytic anemia and severe rhabdomyolysis caused by compound heterozygous mutations of the gene for erythrocyte/muscle isozyme of aldolase, ALDOA(Arg303X/Cys338Tyr). Blood. 2004;103(6):2401-3. doi: 10.1182/blood-2003-09-3160.

34. Mamczur P, Gamian A, Kolodziej J, Dziegiel P, Rakus D. Nuclear localization of aldolase A correlates with cell proliferation. Biochim Biophys Acta. 2013;1833(12):28122822. doi: 10.1016/j.bbamcr.2013.07.013.

35. Ahn BY, Song ES, Cho YJ, Kwon OW, Kim JK, Lee NG. Identification of an anti-aldolase autoantibody as a diagnostic marker for diabetic retinopathy by immunoproteomic analysis. Proteomics. 2006;6(4):12001209. doi: 10.1002/pmic.200500457.

36. Itoh $\mathrm{H}$, Komatsuda A, Ohtani $\mathrm{H}$, et al. Mammalian HSP60 is quickly sorted into the mitochondria under conditions of dehydration. Eur J Biochem 2002;269:5931-5938.

37. Karnati R, Laurie DE, Laurie GW. Lacritin and the tear proteome as natural replacement therapy for dry eye. Exp Eye Res. 2013;117:39-52. doi: 10.1016/j.exer.2013.05.020.

38. Ganguly A, Shields CL. Differential gene expression profile of retinoblastoma compared to normal retina. Mol Vis. 2010;16:1292-1303.

39. Colotti C, Cavallini G, Vitale RL, et al. Effects of aging and anti-aging caloric restrictions on carbonyl and heat shock protein levels and expression. Biogerontology. 2005;6(6):397-406. doi: 10.1007/s10522-005-4906-z.

40. Tsujino S, Shanske S, Sakoda S, Fenichel G, DiMauro S. The molecular genetic basis of muscle phosphoglycerate mutase (PGAM) deficiency. Am J Hum Genet. 1993;52(3):472-477.

41. Jiang X, Sun Q, Li H, Li K, Ren X. The role of phosphoglycerate mutase 1 in tumor aerobic glycolysis and its potential therapeutic implications. Int J Cancer. 2014;135(9):1991-1996. doi: 10.1002/ijc.28637.

42. Santen RJ, Song RX, McPherson R, et al. The role of mitogen-activated protein (MAP) kinase in breast cancer. J Steroid Biochem Mol Biol. 2002;80(2):239-256.

43. Kawashima I, Mitsumori T, Nozaki Y, et al. Negative regulation of the LKB1/AMPK pathway by ERK in human acute myeloid leukemia cells. Exp Hematol. 2015;43(7):524533.e1. doi: 10.1016/j.exphem.2015.03.005.

44. Iida $\mathrm{M}$, Towatari $\mathrm{M}$, Nakao A, et al. Lack of constitutive activation of MAP kinase pathway in human acute myeloid leukemia cells with N-Ras mutation. Leukemia. 1999;13(4):585-589.

45. Ye X, Xu G, Chang Q, et al. ERK1/2 signaling pathways involved in VEGF release in diabetic rat retina. Invest Ophthalmol Vis Sci. 2010;51(10):5226-5233. doi: 10.1167/ iovs.09-4899.

46. Kraus C, Liehr T, Hulsken J, et al. Localization of the human beta-catenin gene (CTNNB1) to 3p21: a region implicated in tumor development. Genomics. 1994;23(1):272-274. doi: 10.1006/geno.1994.1493.

47. MacDonald BT, Tamai K, He X. Localization of the human $\beta$-catenin gene (CTNNB1) to 3p21: a region implicated in tumor development. Developmental Cell. 2009;17:9-26.

48. Thakur R, Mishra DP. Pharmacological modulation of beta-catenin and its applications in cancer therapy. J Cell Mol Med. 2013;17(4):449-456. doi: 10.1111/jcmm.12033.

49. Garcia-Jimenez C, Garcia-Martinez JM, Chocarro-Calvo A, De la Vieja A. A new link between diabetes and cancer: enhanced WNT/beta-catenin signaling by high glucose. J Mol Endocrinol. 2014;52(1):R51-R66. doi: 10.1530/jme13-0152.

50. Chocarro-Calvo A, Garcia-Martinez JM, Ardila-Gonzalez S, De la Vieja A, Garcia-Jimenez C. Glucose-induced beta-catenin acetylation enhances Wnt signaling in cancer. Mol Cell. 2013;49(3):474-486. doi: 10.1016/j. molcel.2012.11.022.

51. Elghazi L, Gould AP, Weiss AJ, et al. Importance of betaCatenin in glucose and energy homeostasis. Sci Rep. 2012;2:693. doi: 10.1038/srep00693.

52. Zhang B, Zhou KK, Ma JX. Inhibition of connective tissue growth factor overexpression in diabetic retinopathy by SERPINA3K via blocking the WNT/beta-catenin pathway. Diabetes. 2010;59(7):1809-1816. doi: 10.2337/db09-1056.

53. Wilker E, Yaffe MB. 14-3-3 Proteins--a focus on cancer and human disease. J Mol Cell Cardiol. 2004;37(3):633-642. doi: 10.1016/j.yjmcc.2004.04.015.

54. Zhang Y, Li M, Wei L, et al. Differential protein expression in perfusates from metastasized rat livers. Proteome Sci. 2013;11(1):37. doi: 10.1186/1477-5956-11-37.

55. Almeida MB, do Nascimento JL, Herculano AM, CrespoLopez ME. Molecular chaperones: toward new therapeutic tools. Biomed Pharmacother. 2011;65(4):239-243. doi: 10.1016/j.biopha.2011.04.025.

56. Sziksz E, Pap D, Veres G, Fekete A, Tulassay T, Vannay A. Involvement of heat shock proteins in gluten-sensitive enteropathy. World J Gastroenterol. 2014;20(21):64956503. doi: 10.3748/wjg.v20.i21.6495.

57. Brehme M, Voisine C, Rolland T, Wachi S, Soper JH, Zhu $\mathrm{Y}$, et al. A chaperome subnetwork safeguards proteostasis in aging and neurodegenerative disease. Cell Rep. 2014;9(3):1135-1150. doi: 10.1016/j.celrep.2014.09.042.

58. Rocha-Martins M, Njaine B, Silveira MS. Avoiding pitfalls of internal controls: validation of reference genes for analysis by qRT-PCR and Western blot throughout rat retinal development. PLoS One. 2012;7(8):e43028. doi: 10.1371/journal.pone.0043028.

59. Saunders PA, Chalecka-Franaszek E, Chuang DM. Subcellular distribution of glyceraldehyde-3-phosphate dehydrogenase in cerebellar granule cells undergoing cytosine arabinoside-induced apoptosis. J Neurochem. 1997;69(5):1820-1828.

60. Kanwar M,Kowluru RA. Role of glyceraldehyde3-phosphate dehydrogenase in the development and progression of diabetic retinopathy. Diabetes. 2009;58(1):227-234. doi: 10.2337/db08-1025.

61. Chee CS, Chang KM, Loke MF, Angela Loo VP, Subrayan V. Association of potential salivary biomarkers with diabetic retinopathy and its severity in type-2 diabetes mellitus: a proteomic analysis by mass spectrometry. PeerJ. 2016;4:e2022. doi: 10.7717/peerj.2022.

62. Zhu X, Miao X, Wu Y, et al. ENO1 promotes tumor proliferation and cell adhesion mediated drug resistance (CAM-DR) in Non-Hodgkin's Lymphomas. Exp Cell Res. 2015;335(2):216-223. doi: 10.1016/j.yexcr.2015.05.020.

63. Song Y, Luo Q, Long H, et al. Alpha-enolase as a potential cancer prognostic marker promotes cell growth, migration, and invasion in glioma. Mol Cancer. 2014;13:65. doi: 10.1186/1476-4598-13-65.

64. Hsiao KC, Shih NY, Fang HL, et al. Surface alpha-enolase promotes extracellular matrix degradation and tumor metastasis and represents a new therapeutic target. PLoS 
One. 2013;8(7):e69354. doi: 10.1371/journal.pone.0069354.

65. Li B, Sheng M, Xie L, et al. Tear proteomic analysis of patients with type 2 diabetes and dry eye syndrome by twodimensional nano-liquid chromatography coupled with tandem mass spectrometry. Invest Ophthalmol Vis Sci. 2014;55(1):177-86. doi: 10.1167/iovs.13-12080.

66. Du S, Guan Z, Hao L, et al. Fructose-bisphosphate aldolase a is a potential metastasis-associated marker of lung squamous cell carcinoma and promotes lung cell tumorigenesis and migration. PLoS One. 2014;9(1):e85804. doi: 10.1371/journal.pone.0085804.

67. Walther EU, Dichgans M, Maricich SM, et al. Genomic sequences of aldolase C (Zebrin II) direct lacZ expression exclusively in non-neuronal cells of transgenic mice. Proc Natl Acad Sci U S A. 1998;95(5):2615-2620.

68. Van den Enden MK, Nyengaard JR, Ostrow E, Burgan JH, Williamson JR. Elevated glucose levels increase retinal glycolysis and sorbitol pathway metabolism. Implications for diabetic retinopathy. Invest Ophthalmol Vis Sci. 1995;36(8):1675-1685.

69. Krasnov GS, Dmitriev AA, Snezhkina AV, Kudryavtseva AV. Deregulation of glycolysis in cancer: glyceraldehyde3-phosphate dehydrogenase as a therapeutic target. Expert Opin Ther Targets. 2013;17(6):6816-93. doi: 10.1517/14728222.2013.775253.

70. Nicholls C, Pinto AR, Li H, et al. Glyceraldehyde-3phosphate dehydrogenase (GAPDH) induces cancer cell senescence by interacting with telomerase RNA component. Proc Natl Acad Sci U S A. 2012;109(33):13308-13313. doi: 10.1073/pnas.1206672109.

71. Koya D, King GL. Protein kinase C activation and the development of diabetic complications. Diabetes. 1998;47(6):859-866.

72. Aiello LP, Bursell SE, Clermont A, et al. Vascular endothelial growth factor-induced retinal permeability is mediated by protein kinase $\mathrm{C}$ in vivo and suppressed by an orally effective beta-isoform-selective inhibitor. Diabetes. 1997;46(9):1473-1480.

73. Madsen-Bouterse S, Mohammad G, Kowluru RA. Glyceraldehyde-3-phosphate dehydrogenase in retinal microvasculature: implications for the development and progression of diabetic retinopathy. Invest Ophthalmol Vis Sci. 2010;51(3):1765-1772. doi: 10.1167/iovs.09-4171.

74. Reddy VS, Raghu G, Reddy SS, Pasupulati AK, Suryanarayana P, Reddy GB. Response of small heat shock proteins in diabetic rat retina. Invest Ophthalmol Vis Sci. 2013;54(12):7674-7682. doi: 10.1167/iovs.13-12715.

75. Abcouwer SF. Angiogenic factors and cytokines in diabetic retinopathy. J Clin Cell Immunol. 2013;Suppl 1(11). doi: 10.4172/2155-9899.

76. Semeraro F, Cancarini A, dell'Omo R, Rezzola S, Romano MR, Costagliola C. Diabetic retinopathy: vascular and inflammatory disease. J Diabetes Res. 2015;2015:582060. doi: 10.1155/2015/582060.

77. Song MK, Roufogalis BD, Huang TH. Modulation of diabetic retinopathy pathophysiology by natural medicines through PPAR-gamma-related pharmacology. Br J Pharmacol. 2012;165(1):4-19. doi: 10.1111/j.14765381.2011.01411.x.

78. Gray LR, Tompkins SC, Taylor EB. Regulation of pyruvate metabolism and human disease. Cell Mol Life Sci. 2014;71(14):2577-2604. doi: 10.1007/s00018-013-1539-2.

79. Zhou YP, Ostenson CG, Ling ZC, Grill V. Deficiency of pyruvate dehydrogenase activity in pancreatic islets of diabetic GK rats. Endocrinology. 1995;136(8):3546-3551. doi: 10.1210/endo.136.8.7628391.

80. Chambers KT, Leone TC, Sambandam N, et al. Chronic inhibition of pyruvate dehydrogenase in heart triggers an adaptive metabolic response. J Biol Chem. 2011;286(13):11155-11162. doi:10.1074/jbc.M110.217349. 